\title{
Topological Phase Transition in Layered GaS and GaSe
}

\author{
Zhiyong Zhu, Yingchun Cheng, and Udo Schwingenschlögl* \\ Physical Sciences and Engineering Division, King Abdullah University of Science and Technology (KAUST), \\ Thuwal 23955-6900, Kingdom of Saudi Arabia
}

(Received 13 December 2011; published 29 June 2012)

By fully relativistic first principles calculations, we predict that appropriate strain engineering of layered $\mathrm{GaX}(X=\mathrm{S}, \mathrm{Se})$ leads to a new class of three-dimensional topological insulators with an excitation gap of up to $135 \mathrm{meV}$. Our results provide a new perspective on the formation of threedimensional topological insulators. Band inversion can be induced by strain only, without considering any spin-orbit coupling. The latter, however, is indispensable for the formation of local band gaps at the crossing points of the inverted bands. Our study indicates that three-dimensional topological insulators can also be realized in materials which comprise light elements only.

DOI: 10.1103/PhysRevLett.108.266805

Even if no symmetry is broken by smoothly varying material parameters, quantum phase transitions are possible due to changes of the topological order [1,2]. The first example is the integer quantum Hall effect [3], where the magnetic field breaks the time-reversal symmetry. Nonzero Thouless-Kohmoto-Nightingale-den Nijs invariants are used to characterize the topologically nontrivial state [4,5]. Considering spin-orbit coupling (SOC), it is found that insulators with unbroken time-reversal symmetry can be further classified according to the $\mathbb{Z}_{2}$ topological invariant [6-9]. Topological classification of the gapped band structure clarifies that gapless conduction states are enforced at interfaces where the topological invariant changes [1,2]. As vacuum can be viewed as a trivial insulator, conducting edge and surface states are enforced by the combination of time-reversal symmetry and SOC on the boundary of a two-dimensional topological insulator, i.e., a quantum spin Hall insulator, [10-12] and a threedimensional (3D) topological insulator [6,13-17]. The search for new classes of 3D topological insulators is of both fundamental interest and technological significance [18-23].

Bulk GaS and GaSe are layered semiconductors with potential applications in optoelectronic devices [24,25]. The excitation gaps are tunable via either uniaxial or hydrostatic strain according to experiments [26]. Both of them consist of weakly van der Waals bound $X-\mathrm{Ga}-\mathrm{Ga}-X$ $(X=\mathrm{S}, \mathrm{Se})$ slabs. In each slab, every $\mathrm{Ga}$ atom is covalently bound to three $X$ atoms and another Ga atom, giving rise to a trigonal prismatic arrangement. The weak inter-slab interaction allows formation of several polytypes [27], due to the possibility of different stacking sequences of the $X$-Ga-Ga- $X$ slabs along the $c$ direction. While $\mathrm{GaS}$ favors the $\beta$ structure (space group no. 194, $P 6_{3} / m m c, D_{6 h}$ ), the $\epsilon$ structure (space group no. $\left.187, P \overline{6} m 2, D_{3 h}\right)$ is the most common polytype for GaSe, see Figs. 1(a) and 1(b), respectively.

In this Letter, we apply fully relativistic first principles calculations to predict that the layered III-VI semiconductors
PACS numbers: 73.43.Nq, 71.20.Nr, 73.20.At, 85.75.-d

$\beta$-GaS and $\epsilon$-GaSe can be transformed into 3D topological insulators via appropriate strain engineering. The band inversion, which is key to realize the topological insulating state, is induced almost completely by structural variations. This goes beyond all existing 3D topological insulators, where strong SOC related to heavy elements is believed to play a dominant role for the band inversion. We show that the indispensable role of the SOC is rather the opening of local energy gaps at the crossing points caused by the band inversion. In band-inverted $\beta$-GaS and $\epsilon$-GaSe these gaps amount to 25 and $135 \mathrm{meV}$, respectively, which is comparable to the $\mathrm{Bi}_{2} \mathrm{Se}_{3}$ series and to the half-Heusler 3D topological insulators. Our results provide a new perspective on the formation of topological insulators and indicate that also materials based on light elements only can be candidates for 3D topological insulators.

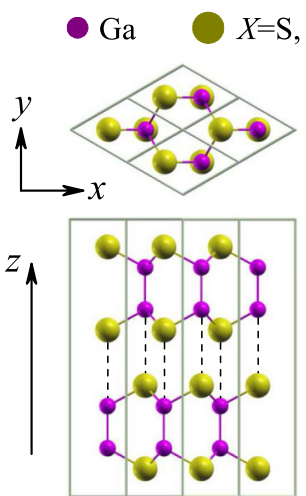

(a)

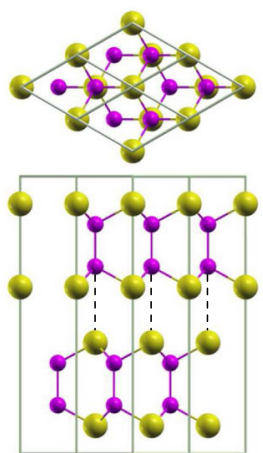

(b)

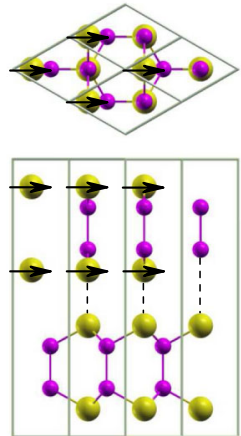

(c)
FIG. 1 (color online). (a) Top and side views of $\beta-\mathrm{Ga} X$ $(X=\mathrm{S}, \mathrm{Se})$. (b) Top and side views of the $\epsilon$ and hypothetical $\epsilon^{\prime}$ structures, which show only slight differences in the atomic environment. (c) Top and side views of the hypothetical $o$ structure, which connects the $\epsilon^{\prime}$ and $\beta$ structures by a smooth variation of the atomic positions along the direction indicated by arrows. See the text for details. Adjacent atoms along the $z$ axis in neighboring slabs are connected by vertical dashed lines. 
For our full potential linearized augmented plane wave calculations we use the WIEN2K package [28], which gives highly accurate results for inhomogeneous structures $[29,30]$. The valence configurations of the $\mathrm{Ga}, \mathrm{S}$, and $\mathrm{Se}$ atoms taken into account in the calculations are $3 d^{10} 4 s^{2} 4 p^{6}, 3 s^{2} 3 p^{6}$, and $3 d^{10} 4 s^{2} 4 p^{6}$, respectively. The same value of $R_{m t}=2.0 \mathrm{bohr}$ is used for the $\mathrm{S}$ and $\mathrm{Se}$ atoms, while 2.2 bohr applies to $\mathrm{Ga}$. We set $R_{m t} K_{\max }=7.0$ and $l_{\max }=10$, and employ a $16 \times 16 \times 3 \mathrm{k}$ mesh and the same exchange correlation functional [31] in all our calculations. The experimental lattice constants are used for the unstrained structure of both $\beta$-GaS ( $a=3.587 \AA$, $c=$ $15.492 \AA)$ [25] and $\epsilon$-GaSe $(a=3.752 \AA, c=15.95 \AA)$ [24]. Strain is simulated by variation of $a$ for constant cell volume. The atomic positions are fully relaxed with a force tolerance of $0.5 \mathrm{mRy} / \mathrm{bohr}$, applying Pulay corrections. The spin-orbit coupling is treated by the secondvariational method with the scalar-relativistic orbitals as basis. States up to 10 Ry above the Fermi energy $\left(E_{F}\right)$ are included in the basis expansion.

The electronic band structure of $\beta$-GaS with SOC is depicted in Fig. 2(a). An indirect band gap is predicted, which agrees with experiments [32]. By increasing the $a / a_{0}$ ratio the conduction states at $\Gamma$ just above $E_{F}$ are lowered in energy, yielding a significantly reduced size of the energy gap $E_{g}(\Gamma)$, see the inset in Fig. 2(a). An indirectto-direct band gap transition occurs, with both the valence band maximum and conduction band minimum located at $\Gamma$. Most interestingly, two bands with $\Gamma_{8+}$ and $\Gamma_{8_{-}}$
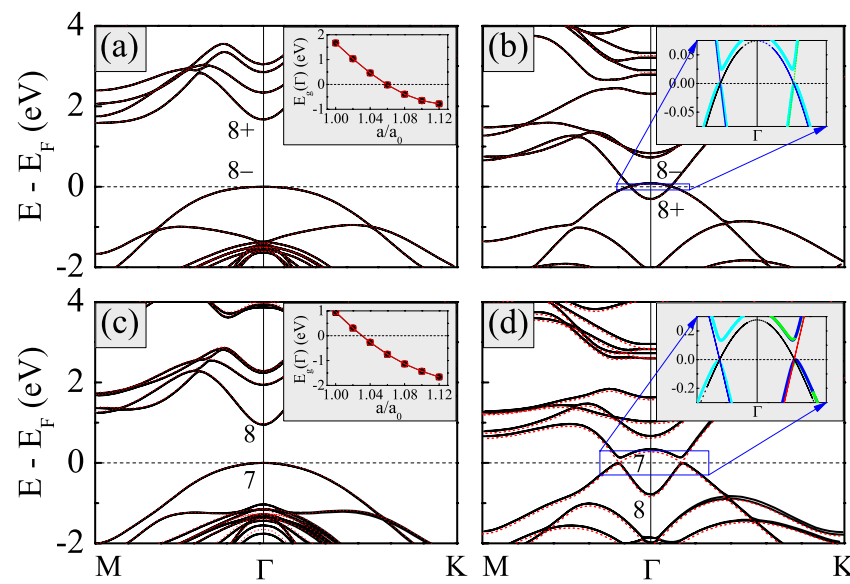

FIG. 2 (color online). (a) Electronic band structures of unstrained $\beta-\mathrm{GaS}$ with (solid lines) and without (dotted lines) SOC. The inset addresses the strain dependent energy gap $E_{g}(\Gamma)$ with (solid squares) and without (open circles) SOC as a function of the $a / a_{0}$ ratio. (b) Electronic band structures of band-inverted $\beta$-GaS $\left(a / a_{0}=1.08\right)$ with (solid lines) and without (dotted lines) SOC. Details around $E_{F}$ are shown in the inset, in which states with different symmetry have different colors and bands with and without SOC are indicated by thick solid and thin dotted lines, respectively. (c),(d) Analogous to (a),(b) but for $\epsilon-\mathrm{GaSe}$. symmetries around $E_{F}$ are inverted after a critical $a / a_{0}$ ratio of $\sim 1.06$. Consequently, an insulating phase with a band gap of $\sim 25 \mathrm{meV}$ is formed, see $\beta$-GaS with $a / a_{0}=$ 1.08 in Fig. 2(b), for example. As observed in well-known 3D topological insulators like $\mathrm{Bi}_{2} \mathrm{Se}_{3}$ [14], inversion of bands with opposite parity is a strong indication of the formation of topologically nontrivial phases. This suggests that the strain induces a topological phase transition in $\beta$-GaS.

To confirm the topological phase transition, all four $\mathbb{Z}_{2}$ topological invariants $\left(\nu_{0} ; \nu_{1} \nu_{2} \nu_{3}\right)$ [6] have to be calculated before and after the band inversion. In $\beta$-GaS, the midpoint of two adjacent $\mathrm{S}$ atoms within neighboring slabs forms an inversion center. Following the method developed by $\mathrm{Fu}$ and Kane [6], the existence of inversion symmetry allows us to calculate the $\mathbb{Z}_{2}$ topological invariants directly from knowledge about the parity of each pair of Kramer's degenerate occupied energy bands at the eight timereversal momenta $(1 \Gamma, 3 M, 1 A$, and $3 L)$. Before the band inversion, the products $\delta_{i}$ of parities are identical, yielding a value of zero for all four $\mathbb{Z}_{2}$ topological invariants, i.e., a topologically trivial state $(0 ; 000)$. When the bands with $\Gamma_{8+}$ and $\Gamma_{8-}$ symmetries switch around $E_{F}$, see Figs. 2(a) and 2(b), $\delta_{\Gamma}$ changes its sign while all other seven $\delta_{i}$ remain unchanged. This gives rise to a topological state with $\mathbb{Z}_{2}$ class $(1 ; 000)$ and confirms that the strain induces a topological phase transition.

Unstrained $\epsilon$-GaSe is also semiconducting, but with a direct band gap located at $\Gamma$, see Fig. 2(c), which agrees with previous calculations [33]. With increasing $a / a_{0}$ ratio, $E_{g}(\Gamma)$ decreases rapidly as in $\beta-\mathrm{GaS}$, compare the inset in Fig. 2(c). After a critical $a / a_{0}$ ratio of $\sim 1.03$, two bands with $\Gamma_{7}$ and $\Gamma_{8}$ symmetries are inverted around $E_{F}$. As a result, an insulating phase with a band gap of $\sim 135 \mathrm{meV}$ is established, see $\epsilon$-GaSe with $a / a_{0}=1.08$ in Fig. 2(d), for example. The band inversion strongly points to a similar strain-engineered topological phase transition as discussed for $\beta$-GaS. In contrast to $\beta$-GaS, however, parity is not defined for $\epsilon$-GaSe, since the inversion symmetry is missing in the $D_{3 h}$ point group. Therefore, the parity-check method of Fu and Kane cannot be applied. An alternative way to determine the $\mathbb{Z}_{2}$ topological nature is a demonstration that an adiabatic transformation between $\epsilon$-GaSe and any other insulator with accessible $\mathbb{Z}_{2}$ topological invariants does not involve an intervening phase transition which closes the band gap at $E_{F}[1,2]$.

We therefore build such a topologically invariant connection between $\epsilon$-GaSe and $\beta$-GaSe. The latter compound has the same symmetry as $\beta$-GaS and the $\mathbb{Z}_{2}$ topological invariants can be calculated. We use bandinverted GaSe with $a / a_{0}=1.08$ as an example to show that the $\epsilon$ structure can be smoothly transformed into its $\beta$ counterpart in three steps. We notice that in $\epsilon$-GaSe the $\mathrm{Ga}$ and $\mathrm{Se}$ atoms in one of the two Se-Ga-Ga-Se slabs are 
inequivalent to those in the other slab. This is due to different interslab atomic environments and leads to slightly different intraslab environments. In a first step towards the $\beta$ structure, the atomic positions in $\epsilon$-GaSe are slightly modified to achieve identical intraslab environments. The resulting hypothetical structure is called $\epsilon^{\prime}$-GaSe. Comparing the crystal structures of the $\beta$ and $\epsilon^{\prime}$ phases, see Figs. 1(a) and 1(b), we find that the $\epsilon^{\prime}$ structure can be transformed into the $\beta$ structure by a $d_{0}=\sqrt{3} a / 3$ shift of the Se atoms in the top slab along the $x$ axis, as shown in Fig. 1(c). Therefore, in a second step, $\epsilon^{\prime}$-GaSe is transformed into hypothetical $o$-GaSe with orthorhombic symmetry (space group no. 38, Amm2) by a fraction of the shift $d_{0}$. In a last step, $o-\mathrm{GaSe}$ then is transformed into $\beta$-GaSe by the full shift $d_{0}$.

The electronic band structures of the hypothetical GaSe phases on the transformation path are shown in Fig. 3. We obtain hardly any change when $\epsilon$-GaSe is transformed into $\epsilon^{\prime}$-GaSe, because the modification of the atomic positions is tiny. The same is true when $\epsilon^{\prime}$-GaSe is transformed into $o$-GaSe, although the crystal symmetry is changed. When the shift is enhanced towards $d_{0}$, the electronic bands of $o$-GaSe are modified slightly near $E_{F}$. Hole pockets are formed at the $\Gamma$ point, see Fig. 3(d), and compensate electron pockets in other regions of the Brillouin zone. However, the topological invariants are not changed as no local gap is closed around $E_{F}$. The change of the crystal symmetry close to $d_{0}$ also hardly affects the electronic band structure.

The above discussion shows that $\epsilon$-GaSe can be smoothly transformed into $\beta$-GaSe without closing the gap around $E_{F}$. We thus can conclude that $\epsilon$-GaSe is topologically equivalent to its $\beta$ counterpart with the same intraslab atomic environment. Because the same parity-check method as used for $\beta$-GaS shows that $\beta$-GaSe is a 3D topological insulator with $\mathbb{Z}_{2}$ class $(1 ; 000)$, this topologically nontrivial

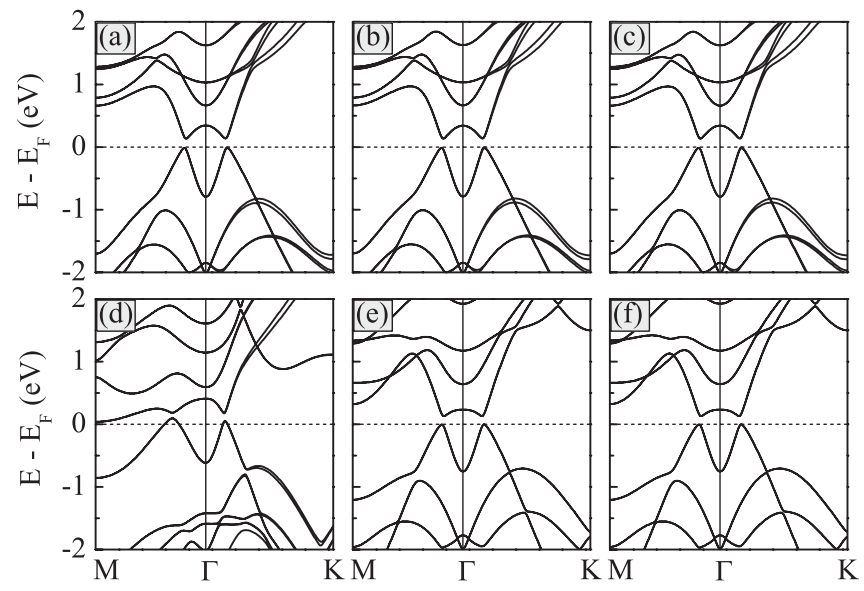

FIG. 3. Fully relativistic electronic band structures of (a) $\epsilon$-GaSe, (b) hypothetical $\epsilon^{\prime}$-GaSe, (c)-(e) hypothetical $o$-GaSe with $d=0.0001 d_{0}, d=0.5 d_{0}$, and $d=0.9999 d_{0}$, respectively, and (f) hypothetical $\beta$-GaSe. See the text for the definition of $d_{0}$. state also applies to $\epsilon$-GaSe. Finally, performing an analogous analysis for $\epsilon$-GaSe before the band inversion results in a trivial insulator. A strain-engineered topological phase transition in $\epsilon$-GaSe therefore is established.

Commonly, SOC is assumed to be the driving force for the band inversion and, hence, the formation of a topologically nontrivial state, similar to the prototypical 3D topological insulator $\mathrm{Bi}_{2} \mathrm{Se}_{3}$ [14]. Recent efforts towards new materials thus have focused on compounds with very heavy elements that introduce strong SOC [1,2,21,34-40]. To quantify the effect of SOC on the band inversion in $\beta$-GaS and $\epsilon$-GaSe, we temporally switch off the SOC in our calculations so that we have only the pure strain effect. Electronic band structures of both unstrained and strained $\beta$-GaS and $\epsilon$-GaSe without SOC and their dependence on the $a / a_{0}$ ratio are addressed in Fig. 2. We find that a band inversion can be induced just by the strain, without any SOC. Furthermore, the changes of $E_{g}(\Gamma)$ due to the SOC are less than 1.5 and $23.5 \mathrm{meV}$ for $\beta-\mathrm{GaS}$ and $\epsilon$-GaSe, respectively. These values are negligible as compared to the absolute value of $E_{g}(\Gamma)$ [1-2 eV, see the insets in Figs. 2(a) and 2(c)]. In contrast to the existing literature, the topological phase transition in our case predominantly is caused by strain. Note that $\beta$-GaS and $\epsilon$-GaSe consist of light elements only.

At this point, it seems SOC is not at all relevant for the formation of the topologically nontrivial states in $\beta$-GaS and $\epsilon$-GaSe. To clarify the role of SOC, we investigate in detail the near- $E_{F}$ states in band-inverted $\beta$-GaS and $\epsilon$-GaSe, compare the insets in Figs. 2(b) and 2(d). Without SOC, we find band crossings and gap closing along the $M-\Gamma$ and $\Gamma-K$ lines once the bands are inverted. Further analysis shows that the same behavior appears for every point in the $k_{z}=0$ plane. The band crossings can be understood as a result of the symmetry incompatibility of the inverted bands. Taking into account the SOC, symmetry compatibility is realized by the additional time-reversal symmetry, and a gap is opened by electron hybridization. Therefore, bearing in mind that for the band inversion the SOC can be replaced by strain, the essential and indispensable role of the SOC is to ensure opening of local energy gaps at the crossing points of the inverted bands throughout the Brillouin zone. This role is confirmed by the fact that the energy gap at the band crossing points is much larger in $\epsilon$-GaSe than in $\beta$-GaS, due to the stronger $\mathrm{SOC}$ of $\mathrm{Se}$ as compared to $\mathrm{S}$. Our conclusion is also in line with the mathematical argument for the Berry phase in Ref. [6].

To gain further insight into the mechanism of the strain induced band inversion, we study the electronic states of unstrained $\beta-\mathrm{GaS}$ around $E_{F}$ in more detail (the same conclusion applies to $\epsilon$-GaSe). For simplicity, no SOC is considered, compare Figs. 4(a) and 4(b). At the $A$ point doubly degenerate states are found below and above $E_{F}$. Towards the $\Gamma$ point, they split up into $\Gamma_{1}^{+} / \Gamma_{4}^{-}$and $\Gamma_{3}^{+} / \Gamma_{2}^{-}$ 


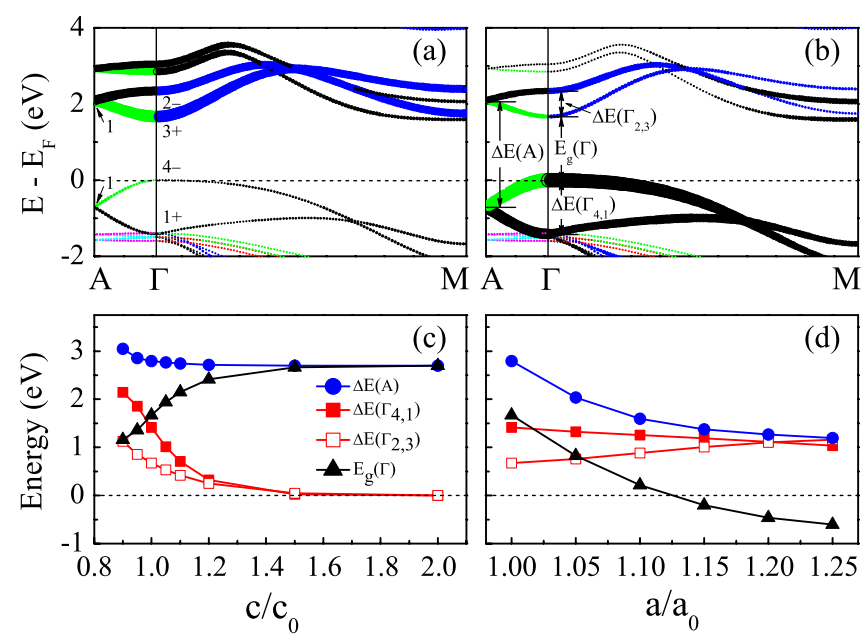

FIG. 4 (color online). (a) Electronic band structure of unstrained $\beta$-GaS without SOC, weighted with the $\mathrm{Ga} / \mathrm{S} s$ character. States with different symmetries are distinguished by different colors. (b) Analogous to (a) but for the $\mathrm{S} p_{z}$ character. (c),(d) $\Delta E(A), \Delta E\left(\Gamma_{4,1}\right), \Delta E\left(\Gamma_{2,3}\right)$, and $E_{g}(\Gamma)$ as functions of the interslab atomic distance $c / c_{0}$ (both $a$ and the slab thickness are fixed) and the intraslab atomic distance $a / a_{0}$ ( $c$ is fixed), respectively.

bands, respectively. As a result, the $\Gamma_{4}^{-}$and $\Gamma_{3}^{+}$bands give rise to the valence band maximum and conduction band minimum, respectively. The relative position of the $\Gamma_{4}^{-}$and $\Gamma_{3}^{+}$bands decides whether a band inversion occurs or not. It also controlls $E_{g}(\Gamma)$, which, according to Fig. 4(b), is determined by the three parameters $\Delta E(A), \Delta E\left(\Gamma_{4,1}\right)$, and $\Delta E\left(\Gamma_{2,3}\right)$.

Figures 4(a) and 4(b) show that the valence $\Gamma_{1}^{+}$and $\Gamma_{4}^{-}$ bands are dominated by the $\mathrm{S} p_{z}$ states, while the conduction $\Gamma_{3}^{+}$and $\Gamma_{2}^{-}$bands are composed of $\mathrm{S} s, \mathrm{~S} p_{z}$, and $\mathrm{Ga} s$ states. Whereas $\Delta E(A)$ is determined by the intraslab covalent $s-p_{z}$ hybridization, $\Delta E\left(\Gamma_{4,1}\right)$ and $\Delta E\left(\Gamma_{2,3}\right)$ are related to interslab van der Waals $p_{z}-p_{z}$ and $s-s$ interactions. This observation is in line with findings for layered transition metal dichalcogenide semiconductors, such as $\mathrm{MoS}_{2}$ [41,42]. We have determined the four parameters $\Delta E(A), \Delta E\left(\Gamma_{4,1}\right), \Delta E\left(\Gamma_{2,3}\right)$, and $E_{g}(\Gamma)$ as functions of the interslab atomic distance $c / c_{0}$ (both $a$ and the slab thickness are fixed) and the intraslab atomic distance $a / a_{0}(c$ is fixed). The results are presented in Figs. 4(c) and 4(d), respectively. For increasing $c / c_{0}, \Delta E(A)$ decreases slightly and approaches a constant value of $2.7 \mathrm{eV}$, as $a$ and the slab thickness are constant. Moreover, $\Delta E\left(\Gamma_{4,1}\right)$ and $\Delta E\left(\Gamma_{2,3}\right)$ decrease rapidly to zero due to the vanishing interslab interaction. Consequently, $E_{g}(\Gamma)$ grows to the value of $\Delta E(A)$. On the other hand, increasing $a / a_{0}$ reduces the intraslab interaction and leads to a strongly reduced $\Delta E(A)$ and slightly altered $\Delta E\left(\Gamma_{4,1}\right)$ and $\Delta E\left(\Gamma_{2,3}\right)$. As a consequence, $E_{g}(\Gamma)$ becomes negative and the bands are inverted. Finally, the interrelation between $a / a_{0}$ and $E_{g}(\Gamma)$ shown in Fig. 2 (fixed cell volume) is explained by the
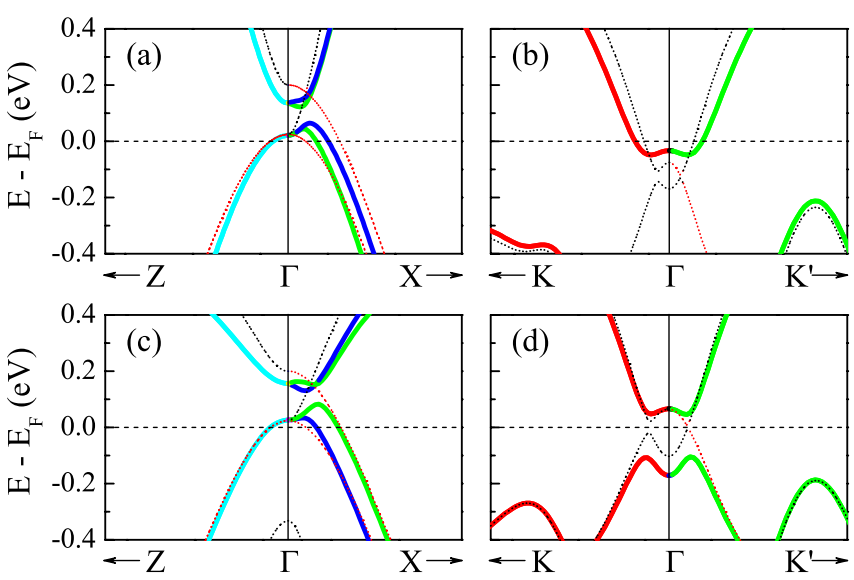

FIG. 5 (color online). Electronic band structures with (thick solid lines) and without (thin dotted lines) SOC for (a) $\mathrm{HgTe}$ with $c=0.95 c_{0}$, (b) $\mathrm{Bi}_{2} \mathrm{Se}_{3}$ with $a=1.03 a_{0}$, (c) PtScBi with $c=0.95 c_{0}$, and (d) $\mathrm{Sb}_{2} \mathrm{Se}_{3}$ with $a=1.03 a_{0}$. States with different symmetries are distinguished by different colors. Hole (electron) pockets at $E_{F}$ compensate electron (hole) pockets in other regions of the Brillouin zone. The topological invariants are not changed as no local gap is closed around $E_{F}$.

strain effect on the strengths of the inter and intraslab electron interactions.

Awareness of the strain effect provides a new perspective on the formation of topological insulators. The first example is HgTe, which is a zero-gap semiconductor with SOCinduced band inversion [2,23]. A slight tetragonal distortion of the crystal structure opens the gap by lifting the degeneracy at the $\Gamma$ point and transforms $\mathrm{HgTe}$ into a topological insulator. Let us study this distortion without considering SOC. We find that band inversion emerges for $c / a \neq 1$. The band gap is closed by symmetry incompatibility along $\Gamma-X$ for $c / a<1$, see Fig. 5(a), and along $\Gamma-Z$ for $c / a>1$ (not shown). Introduction of SOC opens a gap at the band crossing points and a topological insulator is formed. The same mechanism of strain-induced band inversion and SOCinduced opening of the gap also applies to the prototypical $3 \mathrm{D}$ topological insulator $\mathrm{Bi}_{2} \mathrm{Se}_{3}$ [14] and the ternary halfHeusler compound PtScBi [35-37], see Figs. 5(b) and 5(c), respectively. $\mathrm{Sb}_{2} \mathrm{Se}_{3}$, which is predicted to be a trivial insulator [14], can be transformed into a topological insulator by inplane tensile strain, see Fig. 5(d).

In conclusion, we have used fully relativistic first principles calculations to show that the layered semiconductors $\beta$-GaS and $\epsilon$-GaSe can be transformed into 3D topological insulators by strain engineering. As a consequence, we propose to view the formation of topologically nontrivial states from a new perspective: band inversion can result from pure strain due to modifications of the electron hybridizations, without any SOC. SOC, on the other hand, is responsible for introducing a gap at the crossing points of the inverted bands. The SOC-induced energy gaps in band-inverted $\beta$-GaS and $\epsilon$-GaSe amount to 25 and $135 \mathrm{meV}$, respectively, which are typical values known 
from the $\mathrm{Be}_{2} \mathrm{Se}_{3}$ series of compounds. Our data show that 3D topological insulators can also consist of light elements only. Emphasis of the role of strain for the formation of 3D topological insulators is essential from the application point of view. While it is not convenient to tune the SOC by modifying the composition of the sample, strain is an efficient tool to alter the band topology. From the dependence of the total energy on the lattice constants, we estimate that pressures of $10 \mathrm{GPa}(\mathrm{GaS})$ and $4 \mathrm{GPa}$ $(\mathrm{GaSe})$ are sufficient to induce the strain required for a phase transition. Very strong strain can also be induced in thin films via the lattice mismatch with the substrate.

*udo.schwingenschlogl@kaust.edu.sa, +966(0)544700080

[1] M.Z. Hasan and C. L. Kane, Rev. Mod. Phys. 82, 3045 (2010).

[2] X.-L. Qi and S.-C. Zhang, Rev. Mod. Phys. 83, 1057 (2011).

[3] K. von Klitzing, G. Dorda, and M. Pepper, Phys. Rev. Lett. 45, 494 (1980).

[4] R. B. Laughlin, Phys. Rev. B 23, 5632 (1981).

[5] D. J. Thouless, M. Kohmoto, M.P. Nightingale, and M. den Nijs, Phys. Rev. Lett. 49, 405 (1982).

[6] L. Fu and C. L. Kane, Phys. Rev. B 76, 045302 (2007).

[7] L. Fu, C. L. Kane, and E. J. Mele, Phys. Rev. Lett. 98, 106803 (2007).

[8] J.E. Moore and L. Balents, Phys. Rev. B 75, 121306 (2007).

[9] R. Roy, Phys. Rev. B 79, 195322 (2009).

[10] B. A. Bernevig, T. L. Hughes, and S.-C. Zhang, Science 314, 1757 (2006).

[11] A. Roth, C. Brüne, H. Buhmann, L. W. Molenkamp, J. Maciejko, X.-L. Qi, and S.-C. Zhang, Science 325, 294 (2009).

[12] M. König, S. Wiedmann, C. Brüne, A. Roth, H. Buhmann, L. W. Molenkamp, X.-L. Qi, and S.-C. Zhang, Science 318, 766 (2007).

[13] D. Hsieh, D. Qian, L. Wray, Y. Xia, Y. S. Hor, R. J. Cava, and M.Z. Hasan, Nature (London) 452, 970 (2008).

[14] H. Zhang, C.-X. Liu, X.-L. Qi, X. Dai, Z. Fang, and S.-C. Zhang, Nature Phys. 5, 438 (2009).

[15] Y. Xia, D. Qian, D. Hsieh, L. Wray, A. Pal, H. Lin, A. Bansil, D. Grauer, Y. S. Hor, R. J. Cava, and M. Z. Hasan, Nature Phys. 5, 398 (2009).

[16] H.-J. Zhang, C.-X. Liu, X.-L. Qi, X.-Y. Deng, X. Dai, S.C. Zhang, and Z. Fang, Phys. Rev. B 80, 085307 (2009).

[17] Y. L. Chen, J. G. Analytis, J.-H. Chu, Z. K. Liu, S.-K. Mo, X. L. Qi, H. J. Zhang, D. H. Lu, X. Dai, Z. Fang, S.C. Zhang, I. R. Fisher, Z. Hussain, and Z.-X. Shen, Science 325, 178 (2009).
[18] L. Fu and C. L. Kane, Phys. Rev. Lett. 102, 216403 (2009).

[19] X.-L. Qi, R. Li, J. Zang, and S.-C. Zhang, Science 323, 1184 (2009).

[20] A. M. Essin, J.E. Moore, and D. Vanderbilt, Phys. Rev. Lett. 102, 146805 (2009).

[21] M. Dzero, K. Sun, V. Galitski, and P. Coleman, Phys. Rev. Lett. 104, 106408 (2010).

[22] J. Linder, Y. Tanaka, T. Yokoyama, A. Sudbø, and N. Nagaosa, Phys. Rev. Lett. 104, 067001 (2010).

[23] X.-L. Qi and S.-C. Zhang, Phys. Today 63, 33 (2010).

[24] A. Kuhn, A. Chevy, and R. Chevalier, Phys. Status Solidi A 31, 469 (1975).

[25] A. Kuhn, A. Chevy, and R. Chevalier, Acta Crystallogr. Sect. B 32, 983 (1976).

[26] G. L. Belen'kiŭ, E. Y. Salaev, and R. A. Suleŭmanov, Sov. Phys. Usp. 31, 434 (1988).

[27] J. Terhell, V. Brabers, and G. van Egmond, J. Solid State Chem. 41, 97 (1982).

[28] P. Blaha, K. Schwarz, G. K. H. Madsen, D. Kvasnicka, and L. Luitz, WIEN2K An Augmented Plane Wave Plus Local Orbitals Program for Calculating Crystal Properties (Technical University of Vienna, Vienna, 2001).

[29] U. Schwingenschlögl and C. Schuster, Phys. Rev. Lett. 99, 237206 (2007).

[30] U. Schwingenschlögl and C. Schuster, Phys. Rev. Lett. 102, 227002 (2009).

[31] J. P. Perdew, K. Burke, and M. Ernzerhof, Phys. Rev. Lett. 77, 3865 (1996).

[32] E. Aulich, J. L. Brebner, and E. Mooser, Phys. Status Solidi 31, 129 (1969).

[33] D. Errandonea, A. Segura, F. J. Manjón, A. Chevy, E. Machado, G. Tobias, P. Ordejón, and E. Canadell, Phys. Rev. B 71, 125206 (2005).

[34] X. Dai, T. L. Hughes, X.-L. Qi, Z. Fang, and S.-C. Zhang, Phys. Rev. B 77, 125319 (2008).

[35] S. Chadov, X. Qi, J. Kuebler, G. H. Fecher, C. Felser, and S. C. Zhang, Nature Mater. 9, 541 (2010).

[36] H. Lin, L. A. Wray, Y. Xia, S. Xu, S. Jia, R. J. Cava, A. Bansil, and M. Z. Hasan, Nature Mater. 9, 546 (2010).

[37] D. Xiao, Y. Yao, W. Feng, J. Wen, W. Zhu, X.-Q. Chen, G. M. Stocks, and Z. Zhang, Phys. Rev. Lett. 105, 096404 (2010).

[38] B. Yan, C.-X. Liu, H.-J. Zhang, C.-Y. Yam, X.-L. Qi, T. Frauenheim, and C. Zhang, Europhys. Lett. 90, 37002 (2010).

[39] W. Feng, D. Xiao, J. Ding, and Y. Yao, Phys. Rev. Lett. 106, 016402 (2011).

[40] A. Shitade, H. Katsura, J. Kuneš, X.-L. Qi, S.-C. Zhang, and N. Nagaosa, Phys. Rev. Lett. 102, 256403 (2009).

[41] Z. Y. Zhu, Y.C. Cheng, and U. Schwingenschlögl, Phys. Rev. B 84, 153402 (2011).

[42] K. F. Mak, C. Lee, J. Hone, J. Shan, and T. F. Heinz, Phys. Rev. Lett. 105, 136805 (2010). 